\title{
Odontodisplasia Regional y Agenesia de Premolares. Reporte de un Caso
}

\author{
Regional Odontodysplasia and Premolar Agenesis. Case Report.
}

\author{
Gonzalo Martinovic ${ }^{1,2}$; María José Henríquez²; Juan Pablo Alister ${ }^{3}$ \& Carlos Manterola ${ }^{1,3,4}$
}

\begin{abstract}
MARTINOVIC, G.; HENRÍQUEZ, M. J.; ALISTER, J. P. \& MANTEROLA, C. Odontodisplasia regional y agenesia de premolares. Reporte de un caso. Int. J. Odontostomat. 13(3):310-315, 2019.

RESUMEN: La odontodisplasia regional (OR) es una alteración en el desarrollo, no hereditario y que afecta tanto la dentición temporal como la dentición definitiva. Involucra a los tejidos mesodérmicos y ectodérmicos de los dientes lo que es condescendiente con hallazgos clínicos, radiográficos e histológicos. Su etiología aun es desconocida y se presenta mayoritariamente en mujeres. Clínicamente puede afectar al maxilar, a la mandíbula o ambas arcadas pero generalmente solo se ve comprometida una ellas, principalmente el más afectado es el hueso maxilar. Radiográficamente se observa una pobre diferencia entre los tejidos del esmalte y la dentina, siendo tejidos menos radiopacos que su contraparte sana generando un aspecto descrito como "diente fantasma". Histológicamente se observan zonas hipocalcificadas del esmalte con un orden de prismas irregulares mientras que la dentina se observa con un numero reducido de túbulos dentinarios y de consistencia mas fibrosa en su zona coronal. El tratamiento de la OR es controversial ya que su incidencia es baja y la literatura al respecto no es clara. El objetivo de este manuscrito, fue reportar un caso de OR y revisar la literatura relacionada. Presentamos un caso de OR en una paciente de 12 años que presenta ausencia de los dientes $2.4,2.5$ y 2.6; restos radiculares y agenesia de los dientes 3.5 y 4.5. Se describirán sus aspectos clínicos, radiográficos e histológicos. Se realizó una búsqueda sistemática en las siguientes bases de datos: Clínical key, Science Direct, PubMed y SciELO.
\end{abstract}

PALABRAS CLAVE: odontodisplasia; premolares; anormalidades dentales; reporte de casos.

\section{INTRODUCCIÓN}

La odontodisplasia regional (OR) es una rara condición y que afecta tanto la dentición temporal como a la permanente. Presenta un desarrollo anormal, no hereditario de los dientes que afecta tanto el tejido mesodérmico como ectodérmico (Bowden et al., 2018).

Hitchin fue el primero que reconoció esta condición en 1934; pero el crédito se le otorga a McCall \& Wald, quienes fueron los primeros en publicar un reporte de OR y denominarla como "arrested tooth development" en 1947 (Hamdan et al., 2004).

En 1954, se introdujo el termino "shell teeth" por Rushton, quien lo utilizó para describir las características radiográficas de la condición (Bowden et al.); luego, en 1963 se sugirió el término "odontodysplasia" por Zegarelli (Hamdan et al.; Ceballos et al., 2015). Posteriormente, Pindborg en 1970, acuñó el término "regional" (Marques et al., 1999).

La OR afecta a varios dientes adyacentes dentro de un mismo segmento (Hamdan et al.; Babu et al., 2015). Los dientes son de color amarillento-cafesosos o cafés; blandos, hipoplásicos e hipocalcificados; de superficie rugosa debido a un defecto en su mineralización. Adicionalmente a las alteraciones de color y textura del esmalte y dentina el paciente puede desarrollar dolor localizado o inflamación adyacente al área afectada (Hegazi \& Hassan, 2018).

Radiográficamente su aspecto se describe como "ghost teeth"; que se usa para referirse a un diente en

\footnotetext{
${ }^{1}$ Programa de Magíster en Ciencias Médicas, Universidad de La Frontera, Temuco, Chile.

${ }^{2}$ Hospital Militar, Santiago, Chile.

${ }^{3}$ Programa de Doctorado en Ciencias Médicas, Universidad de La Frontera, Temuco, Chile.

${ }^{4}$ Centro de Excelencia en Estudios Morfológicos y Quirúrgicos (CEMyQ), Universidad de La Frontera, Temuco, Chile.
} 
el que la distinción entre esmalte y dentina es difícil de apreciar. Presentan cámaras pulpares excesivamente largas con canales radiculares cortos y ápices abiertos (O Carroll et al., 1991; Hamdan et al.).

Histológicamente se observa un esmalte hipoplásicos, hipomineralizado y enriquecido con matriz de grosor variable. A menudo se han encontrado calcificaciones globulares o "apatitic needles" (Hegazi \& Hassan). La estructura de los primas del esmalte también se observan atípicas.

El tejido dentinario presenta menor grosor, con áreas irregulares de dentina interglobular expandidas. En variados casos la dentina presenta menor número de túbulos dentinarios.

La condición se puede diferenciar de otras alteraciones odontogénicas ya que todos los elementos histológicos del órgano dentario están afectados, mientras que otros dientes en el mismo individuo están normales (Bowden et al.).

Clínicamente los dientes maxilares están doblemente afectados que los dientes mandibulares; principalmente en la zonas anteriores de ambas arcadas. Afecta dientes continuos de un cuadrante en una sola arcada en la mayoría de los casos (Calle,2010); ya que existen presentaciones que afecta más de un cuadrante.

El objetivo de este manuscrito, fue reportar un caso de OR y revisar la literatura relacionada.

\section{PACIENTE Y MÉTODO}

Caso clínico. Paciente 12 años de edad, sexo femenino. Presentó un vano desdentado en la zona maxilar izquierda que databa desde septiembre de 2017, debido a una cirugía a la que fue sometida, de la cual no se poseían registros previos.

Su madre relató que presentaba alteraciones en su fórmula dentaria desde los 3 años de edad; cuando cursó con aumentos de volumen facial, inflamación y dolor local. En esa oportunidad fue diagnosticada como restos radiculares; los que fueron tratados mediante la extracción de éstos. Esta situación fue repetitiva a través de los años, y siempre fue tratada con la extracción de los restos radiculares que iban apareciendo.
En 2017, consultó en otro recinto de salud por dolor en la zona y aumento de volumen, compatible con absceso submucoso; además de restos dentarios que se volvieron a presentar. La madre relató que en esta ocasión el aumento facial era evidente y la profesional que la atendió en aquella consulta solicitó una radiografía panorámica (actualmente extraviada). Con el resultado del examen, sumado a la historia clínica, la paciente fue referida al centro de atención secundario correspondiente donde se realizó la exéresis de los cuerpos extraños nuevamente y se controló hasta el alta. La madre refirió que desconoce respecto de algún estudio histológico de los tejidos extirpados.

La historia médica de la paciente no arrojó antecedentes mórbidos relevantes, sin trauma de la zona, daño local ni radiación.

Los antecedentes médicos, dentales y la historia familiar, no fueron significativos, a excepción de un periodo de hospitalización que sufrió la madre durante el embarazo, alrededor de los 7 meses, por motivos que no recuerda.

Al examen físico extraoral no se observan alteraciones significativas, asimetrías faciales ni lesiones cutáneas vasculares; pero destaca en la zona del párpado superior izquierdo una lesión de aproximadamente $2 \mathrm{~mm}$ de diámetro y $1 \mathrm{~mm}$ de altura, de base sésil, consistencia gomosa y superficie erosionada y umbilicada con zonas descamativas (Fig. 1).

La madre relató que había presentado cuadros en los que esta lesión ha producido secreción de consistencia líquida. Es totalmente asintomática y actual-

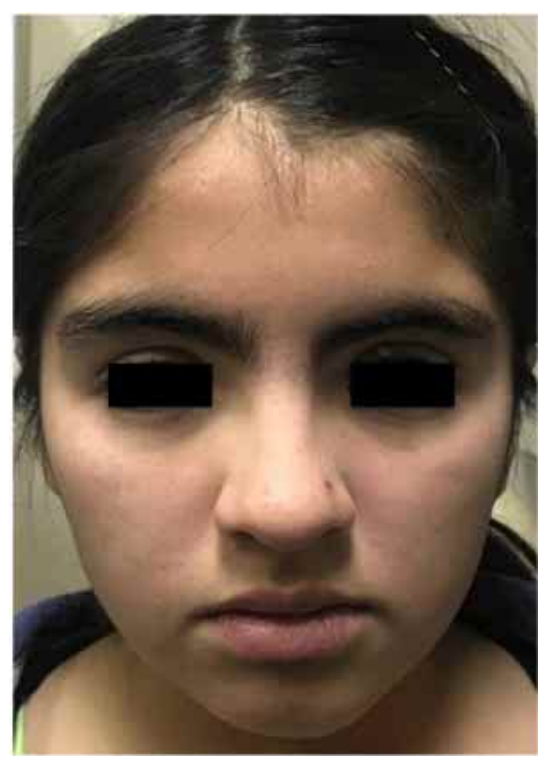

Fig. 1. No se observan alteraciones significativas. No existen asimetrías faciales ni lesiones cutáneas vasculares. Sin embargo, destaca en el párpado superior del ojo izquierdo, una lesión de aproximadamente $2 \mathrm{~mm}$ de diámetro mayor, de base sésil, consistencia gomosa y de superficie erosionada y umbilicada con zonas descamativas. 
mente está siendo evaluada por el oftalmólogo, pues se ha asociado a un cuadro de disminución de la agudeza visual del ojo ipsilateral.

Al examen intraoral, se observó dentición mixta, ausencia de los dientes 2.4, 2.5, 2.6; un cordón mucoso más fibroso, levemente hiperémico e irregular. El reborde alveolar se apreciaba más adelgazado que en su contraparte sana, con una zona de colapso vestibular.

Se destacó un tejido de densidad similar al dentario, compatible con resto radicular del diente 6.4 que generaba dolor a la percusión. Los dientes 5.3 y 6.3 presentaban un aspecto más amarillento-cafesoso, hipoplásico y blando al sondaje.

Además se observó, que los dientes 8.5 y 7.5 no tenían signos de movilidad. El resto del examen no presentaba alteraciones y se encontraba libre de caries, con buena higiene oral.

Frente a estos antecedentes se solicitó radiografía periapical de la zona maxilar izquierda; la cual permitió objetivar ausencia de los dientes 2.4, 2.5, 2.6 y 2.7 ; con cierre apical incompleto y cámara pulpar de aspecto elongado (Fig. 2). Se observó además, una formación similar a un germen dentario sin distinción de tejidos entre esmalte, dentina; y con formación de una cámara pulpar en las cercanías del diente 6.3.

En cercanías del diente 2.7 se verificó otra formación más amorfa, de similar consistencia. Y por último, en proximidad a la mucosa del reborde se constató un tejido de menor tamaño compatible con el resto dentario observado al examen intraoral.

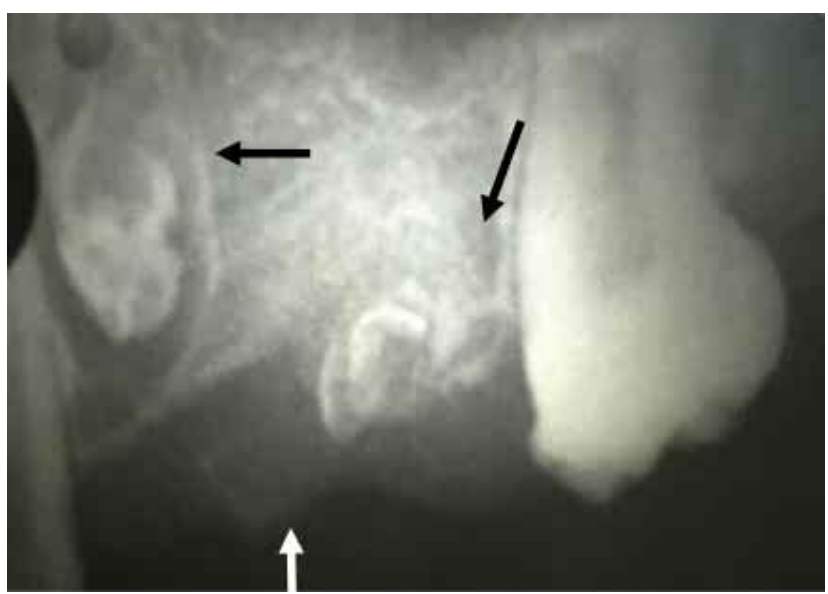

Fig. 2. Radiografía periapical de la zona maxilar izquierda, en la que se puede verificar ausencia de los dientes 2.4, 2.5, 2.6 y diente 2.7 ; con cierre apical incompleto y cámara pulpar de aspecto elongado.
La paciente comentó que los cuadros descritos con antelación siempre comenzaban de esta forma. Con restos múltiples que van apareciendo a nivel gingival y molestias locales durante la masticación.

Dados los antecedentes antes señalados, se solicitó radiografía panorámica y tomografía computada de haz cónico de la zona maxilar izquierda.

En la radiografía panorámica se informó una imagen de densidad mixta heterogénea, con áreas de densidad similar a tejido dentario a nivel de los dientes $2.3,2.4,2.5,2.6$. No se verificó desarrollo de los dientes 4.5 y 3.5. Diente 8.5, 7.5. Persistencia de diente temporal.

Por otra parte, en la tomografía computada de haz cónico se informó zona de diente 2.6; imagen de densidad mixta similar a tejido dentario con halo hipodenso, compatible con estructura dentaria en formación. Tejido óseo próximo a la lesión con amplios espacios medulares y escasas trabéculas óseas, compatible con hueso en hipofunción.

Se planteó entonces la hipótesis diagnóstica de OR más agenesia de los dientes 4.5 y 3.5 .

Se planteó a la madre el diagnóstico y como plan de tratamiento; se ofreció el retiro de estas formaciones dentales anormales, para su envío a estudio histopatológico y confirmación diagnóstica definitiva.

En quirófano ambulatorio y con anestesia local infiltrativa de lidocaina al $2 \%$ y epinefrina 1:100.000; vestibular, palatina y supracrestal, se comenzó el acceso quirúrgico. Se realizó un abordaje lineal, supracrestal con descarga mesial y colgajo de espesor total. El tejido blando era de consistencia muy firme y fibroso. Se realizó decolado para iniciar la osteotomía, en busca de las formaciones dentarias anormales. Se continuó con la toma de muestra de las tres formaciones principales que se observan en los estudios imagenológicos. Finalmente, se reposicionaron los tejidos, con sutura Vicryl 4-0.

La paciente fue controlada a los 4 días, verificándose un tejido en etapa de cicatrización, sin signos de infección e inflamación correspondiente al proceso. La línea de sutura se encontraba en buenas condiciones y la higiene oral era satisfactoria. La paciente relataba que el dolor había ido en disminución desde el día de la cirugía en adelante, con evolución favorable. 
La histopatología del espécimen extirpado fue de OR, describiendo la lesión como mucosa con epitelio plano pluriestratificado, que inmediatamente bajo él presentaba numerosas formaciones redondas, basófilas, calcificadas, con estructura similar a anillos de Liesegang, cerca de islotes o cordones de epitelio odontogénico y entre manojos de tejido fibroblástico. Se informó además, que se observó dicho tejido con calcificaciones hasta el margen quirúrgico.

Se observo tejido con calcificaciones hasta el margen quirúrgico. Este tejido observado aparentemente seria por alteración en la formación de las estructuras dentarías normales.

Frente al diagnostico de OR, se informó a la madre respecto de las posibilidades de tratamiento, que van desde el uso de aparatos removibles para mejorar estética y función; hasta la opción de implantes óseo-integrados una vez terminada su época de crecimiento y desarrollo. La paciente descartó la opción de prótesis removible dado que se encontraba totalmente adaptada a esta situación, y tanto la estética como la función no le afectaban, pues toda su vida la ha desarrollado sin estas dientes.

Se realizó una búsqueda sistemática en las siguientes bases de datos: Clinical key, Science Direct, PubMed y SciELO; utilizando para ello, los términos odontodisplasia regional, etiología, tratamiento, diagnostico diferenciales, histología. No se aplicó filtro de tiempo por la poca prevalencia de la alteración.

\section{DISCUSIÓN}

La OR, es un desarrollo morfológico anormal no hereditario, extraño, que afecta los tejidos duros del diente derivado desde el epitelio y el mesénquima (esmalte, dentina y cemento) (Marques et al.). Afecta tanto en dentición primaria y secundaria. Es comúnmente diagnosticado en la infancia o en la adolescencia entre los 4 y 23 años (Bowden et al.). Presenta predilección por el género femenino sobre el masculino 1,4:1 (Hamdan et al.), sin preferencias étnicas. Afecta más frecuentemente el maxilar (2:1) sobre la mandíbula y generalmente el lado izquierdo (Bowden et al.); y a un sólo cuadrante; a pesar que existe evidencia de casos en los que se han afectado más cuadrantes; o a la zona anterior cruzando la línea media. Suele afectar uno o más dientes del cuadrante de manera secuencial.
El motivo de consulta más frecuente es el retraso en la erupción dentaria o ausencia de estos y la inflamación gingival o sobre crecimiento alrededor de los dientes afectados (Kolenc Fusé, 2004).

Dentro de los diagnósticos diferenciales se ha de considerar: odontodisplasia maxilar segmentaria, displasia hemimaxilar, displasia fibrosa, hiperplasia inflamatoria de la encía, hipertrofia hemifacial, amelogenesis imperfecta, dentinogenesis imperfecta, y displasia dentinaria tipo I y II; pero estas tienden a afectar toda la dentadura o a más de un sólo cuadrante (Hamdan et al.).

No se dispone de datos de prevalencia de la OR, posiblemente como resultado del sub-diagnóstico, perdida de documentación y falta de publicación. Crawford y Aldred presentaron una evaluación estadística en 1989; y uno de los problemas encontrados, fue la evaluación estadística insuficiente de los casos reportados (Tervonen et al., 2004).

Tal como en el presente caso, no siempre están disponibles todos los detalles de la historia clínica, y frecuentemente existen escasos exámenes radiográficos desde los inicios del cuadro. Por otra parte, no siempre se realizan estudios histológicos de los tejidos extirpados a tempranas edades debido a la falta de asociación del cuadro clínico con el diagnóstico.

Si bien la etiología permanece desconocida, se han descrito varias hipótesis que parecen ser el resultado de alteraciones locales que afectan a la formación de los tejidos dentarios durante su desarrollo (CaIle; Bowden et al.) tales como: trauma local, desnutrición, isquemia, medicamentos tomados durante el embarazado, infección local, anormalidades metabólicas, fallas de la migración de las células de la cresta neutral desordenes circulatorios locales, irradiación, daño neurológico, enfermedades sistemas tales como hiperpirexia, factor Rh incompatible, genéticas y drogas teratogénicas (Kolenc Fusé; Calle; Bowden et al.). La OR también se ha asociado a hipofosfatasia, hipocalcemia, presencia de nevus, e hidrocefalia (Ceballos et al.); pero la mayoría de los casos no presenta información relevante, tal como en el reporte actual.

Courson et al., plantearon un desbalance de metaloproteinasas; que son las enzimas que juegan un rol en el desarrollo dentario, y que pueden podrían generar desorganizaciones estructurales, que han sido vistas en OR. 
En este caso, la desorganización estructural de los dientes afectados se han asociado con un desequilibrio en las cantidades de metaloproteinasa de matriz (MMP 1, 2 y 9) y sus inhibidores naturales (TIMP 1 y 2 ) en el tejido gingival alrededor del folículo. Las disminuciones en la red de colágeno parecen favorecer los efectos morfogenéticos perjudiciales en el desarrollo del tejido dental en la OR (Hamdan et al.; Jahanimoghadam et al., 2016).

Generalmente sugerencias de tratamiento protocolizado para OR no pueden ser dadas debido a la necesidad independiente de cada paciente por el nivel de expresión del cuadro que tengan, por las necesidades del paciente ya sean estéticas, funcionales o por las expectativas de este. Dentro de los tratamiento descritos se barajan distintas opciones que generan controversia; tales como la opción quirúrgica o conservadora; es decir remoción de los tejidos alterados o su mantención. Sin embargo, con el fin de no alterar el desarrollo óseo de los maxilares se ha descrito la permanencia del diente erupcionado pero afectado por la OR y posteriormente realizar la restauración de este según sus necesidades.

Por otro lado existe la opción de remover los tejidos afectados no erupcionados seguido por un autotrasplante de gérmenes dentarios o tratamiento de ortodoncia ha sido propuesto. El uso de implantes endoseos no se considera el estándar de referencia, debido a la necesidad de esperar el término de la pubertad y el crecimiento. Por esta razón la utilización de prótesis removibles ha sido sugerida como solución estética y funcional en tempranas edades considerando que generalmente la OR se presenta mayoritariamente en la primera década de vida (Bowden et al.). Siempre la mejor opción será individualizar el manejo de este cuadro según la necesidad del paciente.

A modo de conclusión, se puede señalar que a través del examen y la revisión de la historia clínica de la paciente fue imposible asociar una causa al diagnóstico, debido al me desconocimiento por parte de los padres sobre la historia previa de la paciente, la perdida de exámenes complementarios y la ausencia de documentaciones en instituciones en las cuales recibió atenciones. Además, dentro de los datos actuales no existe ningún antecedente relevante; en vista que el examen oftalmológico por su lesión en el párpado superior izquierdo resulto ser diagnosticado como nevus palpebral. Además de una malformación congénita de la córnea con disminu- ción de la agudeza visual de menos 05 del ojo ipsilateral. En cuanto al tratamiento la opción quirúrgica, se decidió principalmente debido a los problemas que le generaban a la paciente los constantes tejidos dentarios que iban erupcionando periódicamente con mucho dolor, inflamación y cuadros infecciosos a repetición. La paciente rechazó el tratamiento con prótesis removibles debido a que se encuentra adaptada a esta situación y actualmente no le genera problemas estéticos; y la madre prefiere optar por implantes osteointegrados una vez terminada su etapa de desarrollo y crecimiento.

Este caso aporta más información para seguir realizando investigación sobre esta extraña patología para lograr generar mayor conocimiento en cuanto a su aspecto clínico, radiográfico e histológico, otorgando información que evite el mal diagnóstico del cuadro.

MARTINOVIC, G.; HENRÍQUEZ, M. J.; ALISTER, J. P. \& MANTEROLA, C. Regional odontodysplasia and agenesis of premolars. Case report. Int. J. Odontostomat., 13(3):310$315,2019$.

ABSTRACT: Regional odontodysplasia (RO) is a variation in the development; it is not hereditary and it affects both deciduous and permanent dentition. It involves the mesodermal and ectodermal tissues of dental pieces, and coincides with clinical, radiographic and histological findings. Its etiology is still unknown and it reportedly occurs mostly in women. Clinically it can affect the maxilla, mandible or both arches but generally only one is compromised, mainly the maxilla which is affected the most. Radiographically there is limited difference between enamel and dentin tissue, which is less radiopaque than their healthy counterpart, generating an aspect described as "phantom tooth". Histologically hypocalcified areas of the enamel are observed with an irregular order of prisms while the dentine is observed with a reduced number of dentinal tubules and more fibrous consistency in the coronal area. RO treatment is controversial since its incidence is low and the literature on these events is not clear. The aim of this manuscript was to report a case of $\mathrm{RO}$ and review related literature. We present a case of RO in a 12-year-old patient who presents absence of parts 2.4.2.5 and 2.6; radicular remains and agenesis of parts 3.5 and 4.5 . Its clinical, radiographic and histological aspects are described. A systematic search was carried out in the following databases: Clinical key, Science Direct, PubMed and SciELO.

KEY WORDS: "Odontodysplasia"[Mesh]; "Odontodysplasia/therapy" [Mesh]; "Bicuspid"[Mesh]; "Tooth Abnormalities"[Mesh]; "Amelogenesis Imperfecta"[Mesh]; "Case Reports" [Publication Type]. 


\section{REFERENCIAS BIBLIOGRÁFICAS}

Babu, N. S.; Smriti, R. J. \& Pratima, D. B. Regional odontodysplasia: Report of an unusual case involving mandibular arch. Contemp. Clin. Dent., 6(2):237-9, 2015.

Bowden, E.; Carroll, C.; Gill, M. \& Llewelyn, R. Cervicofacial infection in a 3-year-old child with regional odontodysplasia: A case report and literature review. J. Oral Maxillofac. Pathol., 22(Suppl. 1):S117-S120, 2018.

Calle, M. A. Odontodisplasia regional: Manejo endodóntico. Rev. CES Odontol., 23(2):67-72, 2010.

Ceballos, O. D. M.; Espinal, G. E. \& Jones, M. Development abnormalities and dental training: odontodysplasia. Int. J. Odontostomat., 9(1):129-36, 2015.

Hamdan, M. A.; Sawair, F. A.; Rajab, L. D.; Hamdan, A. M. \& AlOmari, I. K. Regional odontodysplasia: a review of the literature and report of a case. Int. J. Paediatr. Dent., 14(5):363-70, 2004.

Hegazi, F. \& Hassan, M. Regional odontodysplasia crossing the midline. J. Dent. Child. (Chic.), 85(2):88-91, 2018.

Jahanimoghadam, F.; Pishbin, L. \& Rad, M. Clinical, radiographic, and histologic evaluation of regional odontodysplasia: a case report with 5-year follow-up. J. Dent. (Shiraz), 17(2):159-63, 2016.

Kolenc Fusé, F. J. Agenesias dentarias: en busca de las alteraciones genéticas responsables de la falta de desarrollo. Med. Oral Patol. Oral Cir. Bucal., 9(5):385-95, 2004.

Marques, A. C.; Castro, W. H. \& do Carmo, M. A. Regional odontodysplasia: an unusual case with a conservative approach. Br. Dent. J., 186(10):522-4, 1999.

Metin, M.; Üçok, C.; Keskin, A. \& Çizmeci, F. Regional odontodisplasia presenting as a soft tissue swelling. Tr. J. Med. Sci., 29:715-7, 1999.

O Carroll, M. K.; Duncan, W. K. \& Perkins, T. M. Dentin dysplasia: review of the literature and a proposed subclassification based on radiographic findings. Oral Surg. Oral Med. Oral Pathol., 72(1):119-25, 1991.

Tervonen, S. A.; Stratmann, U.; Mokrys, K. \& Reichart, P. A. Regional odontodysplasia: a review of the literature and report of four cases. Clin. Oral Investig., 8(2):45-51, 2004.
Dirección para correspondencia:
Dr. Carlos Manterola

Centro de Excelencia en Estudios Morfológicos y Quirúrgi$\cos ($ CEMyQ)

Universidad de La Frontera

Temuco

CHILE

Email: carlos.manterola@ufrontera.cl

Recibido : 27-03-2019

Aceptado: 28-04-2019 